\title{
Graviton scattering amplitudes in $M$ theory
}

\author{
Katrin Becker* and Melanie Becker ${ }^{\dagger}$ \\ California Institute of Technology 452-48, Pasadena, California 91125 \\ (Received 5 January 1998; published 13 April 1998)
}

\begin{abstract}
We compute graviton scattering amplitudes in $\mathrm{M}$ theory using Feynman rules for a scalar particle coupled to gravity in eleven dimensions. The processes that we consider describe the single graviton exchange and the double graviton exchange, that in $\mathrm{M}$ (atrix) theory correspond to the $v^{4} / r^{7}$ and $v^{6} / r^{14}$ term, respectively. We argue that the $v^{6} / r^{14}$ term appearing in $\mathrm{M}$ (atrix) theory at two loops can be obtained from the covariant eleven-dimensional four-graviton amplitude. Finally, we calculate the $v^{8} / r^{18}$ term appearing at two loops in $\mathrm{M}$ (atrix) theory. It has been previously conjectured that this term is related to a four graviton scattering amplitude involving the $R^{4}$ vertex of M theory.
\end{abstract}

[S0556-2821(98)01310-1]

PACS number(s): 11.25.Sq, 04.65.+e

\section{INTRODUCTION}

$\mathrm{M}$ theory is the "quantum version" of elevendimensional supergravity. The low energy degrees of freedom and interactions of this theory are those of general relativity. However, if one wants to calculate, e.g., radiative corrections to graviton scattering amplitudes one has to know how to describe the short distance degrees of freedom of $M$ theory. $M$ (atrix) theory [1] is a proposal that in the infinite momentum frame these degrees of freedom can be described in terms of Dirichlet zero branes. Graviton scattering amplitudes that are usually hard to compute (or even infinite) using standard quantum field theory techniques can be calculated in terms of a simple quantum mechanical model. So for example, the $v^{4} / r^{7}$ term appearing at one-loop in $\mathrm{M}$ (atrix) theory has an interpretation as a single graviton exchange diagram in eleven dimensions, while the $v^{6} / r^{14}$ term appearing at two-loops in $\mathrm{M}$ (atrix) theory describes a correction coming from general relativity [2]. An example of a quantum gravity correction is the $v^{8} / r^{18}$ term appearing at two loops in M(atrix) theory [3].

In this paper we would like to consider these three terms in the effective potential between two gravitons in some detail. In Sec. II we will compute the $v^{4} / r^{7}$ and $v^{6} / r^{14}$ terms using Feynman rules for supergravity. In Sec. III we will show that the relativistic correction can be calculated using the covariant four-graviton amplitude in eleven dimensions [4,5]. In Sec. IV we will make some remarks about quantum gravity corrections and give the exact result for the $v^{8}$ term appearing in M(atrix) theory at two loops. The Appendix contains more explicitly some of our calculations.

\section{SINGLE GRAVITON EXCHANGE}

In this section we would like to consider the scattering of two gravitons in eleven dimensions. The Feynman rules that we need to do this calculation were derived a long time ago by Feynman [6] and De Witt [7] (see also [8]). The vertices involving gravitons are rather complicated so that we will

\footnotetext{
*Email address: beckerk@ theory.caltech.edu

†Email address: mbecker@theory.caltech.edu
}

address the problem using a simpler model that captures the important properties of the calculation. First, we will be assuming that one of the gravitons involved in the process is heavy so that it can be treated as the source for the gravitational field in which the other graviton is scattered. Therefore, we will be considering a problem of potential scattering. Our second assumption is that we can treat the "probe graviton" as a scalar particle. The vertices describing the coupling of a scalar to gravity are simpler than vertices involving gravitons (see [7]).

We consider the eleven-dimensional ${ }^{1}$ Einstein action coupled to a massless scalar field

$$
S=\int d^{11} x \sqrt{-g}\left(\frac{1}{\kappa_{11}^{2}} R+\frac{1}{2} g_{\mu \nu} \partial^{\mu} \varphi \partial^{\nu} \varphi\right),
$$

and we expand the metric around a flat Minkowski background

$$
g_{\mu \nu}=\eta_{\mu \nu}+h_{\mu \nu}
$$

Here $h_{\mu \nu}$ is a small fluctuation that describes the source graviton field. The action for the "probe graviton'" $\varphi$ then becomes

$$
\begin{aligned}
S_{\varphi}= & \int d^{11} x\left(-\frac{1}{2} \varphi \square \varphi+\frac{1}{2} h_{\mu \nu}\right. \\
& \left.\times\left[\partial^{\mu} \varphi \partial^{\nu} \varphi-\frac{1}{2} \eta^{\mu \nu} \partial_{\lambda} \varphi \partial^{\lambda} \varphi\right]+{ }^{\prime} \cdot h h \varphi \varphi,\right),
\end{aligned}
$$

where we have indicated explicitly the cubic vertex and only symbolically the quartic vertex whose explicit form can be found in [7]. The source graviton has vanishing transverse velocity and it moves with the speed of light in the eleven direction [2]. It is described by the Aichelburg-Sexl metric [9] whose only nonvanishing component is $h_{--}$:

$$
h_{--}=\frac{15 N_{s}}{2 R^{2} M_{\mathrm{Pl}}^{9}} \frac{1}{r^{7}},
$$

\footnotetext{
${ }^{1} \mathrm{We}$ are using the signature $(-,+, \ldots,+)$.
} 
where $p_{-}^{s}=N_{s} / R$ is the momentum of the source graviton and $M_{\mathrm{Pl}}$ is the Planck mass that is related to the gravitational constant by $\kappa_{11}^{2}=16 \pi^{5} / M_{\mathrm{Pl}}^{9}$. The action takes then the form

$$
S_{\varphi}=\frac{1}{2} \int d^{11} x\left(-\varphi \square \varphi+h^{++} \partial_{+} \varphi \partial_{+} \varphi+\cdots\right) .
$$

In the following we will consider the single graviton exchange process in the potential scattering description. The lightlike compactification which is described in terms of a nonrelativistic Schrödinger equation is relevant for finite $N$ $\mathrm{M}$ (atrix) theory [10]. The spacelike compactification is described in terms of a relativistic Klein-Gordon equation which leads to additional terms in the effective potential that are of no relevance for finite $N$. We will further generalize these results to the double graviton exchange describing the relativistic corrections.

\section{A. One graviton exchange in the lightlike compactification}

In the lightlike compactification the equation of motion for $\varphi$ that follows from (2.5) is

$$
\left(\frac{1}{2 m} \frac{\partial^{2}}{\partial \vec{x}^{2}}+i \frac{\partial}{\partial \tau}\right) \varphi=V \varphi, \quad \text { with } V=-\frac{1}{2 m} h_{--} \partial_{\tau}^{2} .
$$

Here $m=N_{p} / R, \tau=x^{+} / 2$ and we have used that $\varphi$ is in a state of definite $p_{-}$. Equation (2.6) has the form of a nonrelativistic Schrödinger equation for a particle with mass $m$ in an external potential $V$.

The $S$ matrix can be obtained using the standard quantum mechanics techniques. The free propagator for this massive particle is

$$
K_{0}\left(\vec{x}_{f}, \tau_{f} ; \vec{x}_{i}, \tau_{i}\right)=\theta\left(\tau_{f}-\tau_{i}\right) \int \frac{d^{9} \vec{p}}{(2 \pi)^{9}} e^{i \vec{p}\left(\vec{x}_{f}-\vec{x}_{i}\right)-i E\left(\tau_{f}-\tau_{i}\right)},
$$

where $(\vec{x}, \vec{p})$ denotes the nine-dimensional transverse space and $E=\vec{p}^{2} / 2 m$ is the light-cone energy. The plane wave solutions to the equation of motion have a nonrelativistic normalization

$$
\varphi(\vec{x}, \tau)=\frac{1}{(2 \pi)^{9 / 2}} e^{i(\vec{p} \vec{x}-E \tau)} .
$$

To first order the $S$ matrix is given by

$$
\begin{aligned}
S^{(1)}= & -i \int \varphi_{\text {out }}^{*}\left(\vec{x}_{f}, \tau_{f}\right) K_{0}\left(\vec{x}_{f}, \tau_{f} ; \vec{x}, \tau\right) \\
& \times V(\vec{x}, \tau) K_{0}\left(\vec{x}, \tau ; \vec{x}_{i} ; \tau_{i}\right) \varphi_{\text {in }}\left(\vec{x}_{i}, \tau_{i}\right) d \vec{x}_{f} d \vec{x} d \vec{x}_{i} d \tau .
\end{aligned}
$$

After evaluating the different integrals we get $^{2}$

\footnotetext{
${ }^{2}$ Here and in the following we omit the factor $2 \pi i \delta\left(E_{f}-E_{i}\right)$ that expresses energy conservation from all the scattering amplitudes.
}

$$
S^{(1)}=-\frac{N_{s} N_{p}}{R^{3} M_{\mathrm{Pl}}^{9}} \frac{v^{4}}{8(2 \pi)^{5}} \frac{1}{|\vec{k}|^{2}}
$$

where $\vec{k}=\vec{p}_{f}-\vec{p}_{i}$ is the nine-dimensional momentum transfer. The effective potential is obtained by Fouriertransforming

$$
V_{\mathrm{eff}}^{(1)}=-\frac{15}{16} \frac{N_{s} N_{p}}{R^{3} M_{\mathrm{Pl}}^{9}} \frac{v^{4}}{r^{7}}
$$

This is the result that was computed in [1], [11], and [2].

\section{B. One graviton exchange in the spacelike compactification}

The previous calculation can be repeated for a spacelike compactification. In this case the equation of motion for $\varphi$ has the form of a relativistic Klein-Gordon equation:

$$
\left(\frac{\partial^{2}}{\partial \vec{x}^{2}}-\frac{\partial^{2}}{\partial x_{0}^{2}}+m^{2}\right) \varphi=V \varphi, \quad \text { with } V=-h_{--}\left(\partial_{x_{0}}-m\right)^{2},
$$

where $m$ is the eleven-component of the momentum. The solutions to the free equation of motion are now plane waves with a relativistic normalization

$$
\varphi(x)=\frac{1}{(2 \pi)^{9 / 2}} \frac{1}{\sqrt{2 E}} e^{i p x},
$$

where $E^{2}=\vec{p}^{2}+m^{2}$.

The $S$ matrix to first order is now

$$
S^{(1)}=-i \int \varphi_{\text {out }}^{*}(y) V(y) \varphi_{\text {in }}(y) d^{10} y
$$

After performing the integration and a Fourier transformation we obtain

$$
V_{\mathrm{eff}}^{(1)}=-\frac{N_{p}}{R} \frac{h_{--}}{2} \frac{\left(\sqrt{1-v^{2}}-1\right)^{2}}{\sqrt{1-v^{2}}} .
$$

This result agrees with [2]. While in the lightlike compactification the $S$ matrix contained only a $v^{4}$ term (2.11), here we obtain a series in terms of $v$. The difference is due to the fact that in the second calculation $\varphi$ obeys relativistic kinematics, while in the lightlike compactification $\varphi$ obeys a nonrelativistic Schrödinger equation. These additional terms have no relevance for finite $N \mathrm{M}$ (atrix) theory and are subleading in the large $N$ expansion.

\section{GENERAL RELATIVITY CORRECTIONS}

\section{A. Double graviton exchange in the lightlike compactification}

Corrections coming from general relativity are described by the diagonal term in the table appearing in [2]. They have integer powers in $\kappa_{11}$. The simplest example of a correction of this type is the $v^{6} / r^{14}$ term appearing in $\mathrm{M}$ (atrix) theory at two loops. It can be obtained from the double graviton exchange or equivalently a second order scattering process. Here we will evaluate the contribution involving two cubic vertices of the type (2.6). To second order the $S$ matrix described by (2.6) is given by 


$$
\begin{aligned}
S^{(2)}= & -\int \varphi_{\text {out }}^{*}\left(\vec{x}_{f}, \tau_{f}\right) K_{0}\left(\vec{x}_{f}, \tau_{f} ; \vec{x}^{\prime}, \tau^{\prime}\right) V\left(\vec{x}^{\prime}, \tau^{\prime}\right) \\
& \times K_{0}\left(\vec{x}^{\prime}, \tau^{\prime} ; \vec{x}, \tau\right) V(\vec{x}, \tau) K_{0}\left(\vec{x}, \tau ; \vec{x}_{i}, \tau_{i}\right) \\
& \times \varphi_{\text {in }}\left(\vec{x}_{i}, \tau_{i}\right) d \vec{x}_{i} d \vec{x} d \tau d \vec{x}^{\prime} d \tau^{\prime} d \vec{x}_{f}
\end{aligned}
$$

We can evaluate the $\vec{x}_{i}, \vec{x}_{f}, \vec{x}$, and $\vec{x}^{\prime}$ integrals exactly. From the $\tau, \tau^{\prime}$ integrals we get the contribution

$$
\int_{-\infty}^{+\infty} d \tau e^{i \tau\left(E_{f}-E_{i}\right)} \int_{0}^{+\infty} d t e^{i t\left(E_{f}-\vec{p}^{2} / 2 m\right)},
$$

where we have transformed to a new integration variable $t$ $=\tau-\tau^{\prime}$. The first integral again gives energy conservation but the second integral does not converge. We can evaluate it using a trick of Feynman and Hibbs [12]. Expression (3.2) can be written as

$$
2 \pi \delta\left(E_{f}-E_{i}\right) \lim _{\epsilon \rightarrow 0} \frac{-i}{E_{f}-E+i \epsilon},
$$

where $E=\vec{p}^{2} / 2 m$. The $S$ matrix to second order then takes the form

$$
\begin{aligned}
S^{(2)}= & \frac{N_{p}^{3} N_{s}^{2}}{R^{7} M_{\mathrm{Pl}}^{18}} \frac{v^{8}}{32} \lim _{\epsilon \rightarrow 0} \int \frac{d^{9} \vec{p}}{(2 \pi)^{10}} \frac{1}{\left|\vec{p}_{f}-\vec{p}\right|^{2}} \\
& \times \frac{1}{\left|\vec{p}_{i}-\vec{p}\right|^{2}} \frac{1}{\vec{p}^{2}-\vec{p}_{f}^{2}-i \epsilon} .
\end{aligned}
$$

This is a standard integral that appears in the computation of radiative corrections to Coulomb scattering [13] and we solve it explicitly in the Appendix. The leading nonanalytic behavior of this integral is

$$
I=\frac{\pi^{6}}{256} \frac{|\vec{k}|^{5}}{p_{f}^{2}} .
$$

In order to Fourier transform this expression we need

$$
\int d^{9} \vec{k} e^{i \overrightarrow{k r}}|\vec{k}|^{5}=-2^{15} 3^{3} 5^{2} \frac{\pi^{4}}{r^{14}} .
$$

Up to a constant the final result for the effective potential is

$$
V_{\mathrm{eff}}^{(2)} \propto-\frac{225}{64} \frac{N_{s}^{2} N_{p}}{R^{5} M_{\mathrm{Pl}}^{18}} \frac{v^{6}}{r^{14}}
$$

This shows that this diagram indeed gives a contribution to the $v^{6} / r^{14}$ term. To get the exact numerical coefficient one would have to evaluate all possible double graviton exchange diagrams. Moreover, from the Appendix we observe that the imaginary part of this amplitude is infrared divergent. ${ }^{3}$ This is familiar from the calculation of radiative corrections to Coulomb scattering in four dimensions [13]. To cancel this divergence one gives a mass to the photon and the sum over all polarizations gives a finite amplitude. Po-

\footnotetext{
${ }^{3}$ Infrared divergences in quantum gravity can be treated in the same manner as in quantum electrodynamics [14].
}

larizations for the probe graviton will have to be taken into account here as well at some point. This calculation seems much harder because graviton vertices are rather complicated. The easiest way to proceed might be to compute this four-graviton amplitude using type-IIA string theory as we do in the next section. Of course, we expect the loop amplitude to reproduce the result of the classical calculation of [2]. This is similar to the situation appearing in the fourdimensional Newton potential ${ }^{4}$ [15]. Here general relativity corrections can be computed through graviton scattering amplitudes or by a classical calculation in which the zero component of the Schwarzschild metric is expanded.

\section{B. The covariant four-graviton amplitude}

It is curious to see that in the spacelike compactification the general relativity correction of the previous section can be computed from the covariant four-graviton amplitude $[4,5]$. The sum of the tree level and one-loop contributions to the on-shell four-graviton amplitude in $D$ dimensions is given by [4]

(kinematical factor)

$$
\begin{aligned}
& \times\left(\frac{1}{s t u} \frac{\Gamma\left(1-\frac{\alpha^{\prime}}{2} s\right) \Gamma\left(1-\frac{\alpha^{\prime}}{2} t\right) \Gamma\left(1-\frac{\alpha^{\prime}}{2} u\right)}{\Gamma\left(1+\frac{\alpha^{\prime}}{2} s\right) \Gamma\left(1+\frac{\alpha^{\prime}}{2} t\right) \Gamma\left(1+\frac{\alpha^{\prime}}{2} u\right)}\right. \\
& \left.+c_{1} g^{(1)}+\cdots\right),
\end{aligned}
$$

where $c_{1}$ is a calculable number determined by unitarity [16] and $s, t$, and $u$ are the Mandelstam variables

$$
\begin{aligned}
& s=-\left(p_{1}+p_{2}\right)^{2}, \\
& t=-\left(p_{1}+p_{4}\right)^{2}, \\
& u=-\left(p_{1}+p_{3}\right)^{2} .
\end{aligned}
$$

Here 1 and 2 are incoming particles while 4 and 3 are outgoing and $p_{i}$ describe their corresponding momenta. The first term in formula (3.8) represents the tree level contribution while the one-loop part of the amplitude $g^{(1)}$ is given by

$$
g^{(1)}=\frac{\kappa_{10}^{2}}{\alpha^{\prime}} \int \frac{d^{2} \tau}{(\operatorname{Im} \tau)^{2}} F(\tau)\left[F_{2}(a, \tau)\right]^{10-D},
$$

where $a=\sqrt{\alpha^{\prime}} / R$ is a dimensionless parameter. The expression for $F(\tau)$ can be found in [4]. We are interested in the low-energy limit of this amplitude which corresponds to the zero slope limit $\alpha^{\prime} \rightarrow 0$. The one-loop contribution is divergent for $D \geqslant 8$ (here we consider $D=11$ ). The finite part of (3.8) reduces to

\footnotetext{
${ }^{4}$ We thank Steven Weinberg for pointing out this reference to us.
} 


$$
(\text { kinematical factor }) \times\left(\frac{1}{s t u}+c_{1} g_{0}^{(1)}+\cdots\right) \text {, }
$$

where

$$
\begin{aligned}
g_{0}^{(1)}= & \kappa_{D}^{2} c(\gamma)\left[I_{\gamma}(s, t)+I_{\gamma}(t, s)+I_{\gamma}(s, u)\right. \\
& \left.+I_{\gamma}(u, s)+I_{\gamma}(t, u)+I_{\gamma}(u, t)\right],
\end{aligned}
$$

is the asymptotic value of $g^{(1)}$ and $\gamma=D / 2-4$. Here $c(\gamma)$ is a constant determined by the dimensionality of the spacetime:

$$
c(\gamma)=\frac{1}{4}\left(\frac{\pi}{4}\right)^{\gamma+1 / 2}[\sin (\pi \gamma) \Gamma(\gamma+5 / 2)]^{-1} .
$$

The function $I(\gamma)$ is

$$
I_{\gamma}(s, t)=t^{\gamma+1} \int_{0}^{1} \frac{(1-x)^{\gamma+1} d x}{s x-t(1-x)} .
$$

In $D=11$ we get $\gamma=3 / 2$ so that $c(\gamma)$ is

$$
c(3 / 2)=-\frac{\pi^{2}}{384},
$$

and $g_{0}^{(1)}$

$$
\begin{aligned}
g_{0}^{(1)}= & -\frac{\pi^{2}}{384} \kappa_{11}^{2}\left[I_{3 / 2}(s, t)+I_{3 / 2}(t, s)\right. \\
& \left.+I_{3 / 2}(s, u)+I_{3 / 2}(u, s)+I_{3 / 2}(t, u)+I_{3 / 2}(u, t)\right] .
\end{aligned}
$$

The integrals $I_{3 / 2}$ can all be evaluated exactly but give rather complicated functions. Expanding around $t=0$ we are interested in the leading nonanalytic ${ }^{5}$ term in $t$. It is given by

$$
g_{0}^{(1)}=i \kappa_{11}^{2} \frac{\pi^{3}}{384} \frac{t^{5 / 2}}{s}+\mathcal{O}\left(t^{7 / 2}\right) \text {. }
$$

The kinematical factor in (3.8) can be computed from the tree level contribution of this formula. We consider the case where the first graviton has vanishing transverse velocity, i.e., $p_{1}=(M, 0, M)$, where $M=N_{s} / R$ is the mass. The second incoming graviton has $p_{2}=\left(E_{2}, \vec{p}_{2}, m\right)$, where $E_{2}^{2}=\vec{p}_{2}^{2}+m^{2}$ is the relativistic energy, $\vec{p}_{2}$ is the nine-dimensional transverse momentum and $m=N_{p} / R$ is the mass. To leading order in the velocity we have

$$
\begin{aligned}
& s=-M m \vec{v}^{2}+\cdots, \\
& t=-|\vec{k}|^{2}+\cdots, \\
& u=-s-t .
\end{aligned}
$$

Here we have set $\left|\vec{p}_{2}\right|=m v$ to leading order in $v$. As can be easily checked the result for the tree level contribution com-

\footnotetext{
${ }^{5}$ The analytic terms correspond to contact terms after Fourier transforming.
}

puted in [1] and [11] is reproduced with a kinematical factor $-\kappa_{11}^{2} s^{2} u^{2}$. The tree level amplitude then becomes

$$
A_{\text {tree }}=\kappa_{11}^{2} \frac{s^{2}}{t}=-\kappa_{11}^{2} M^{2} m^{2} \frac{v^{4}}{|\vec{k}|^{2}} .
$$

In order to obtain the potential we are interested in the Fourier transformed of this expression with respect to the momentum transfer. Using the relation

$$
\int \frac{d^{9} \vec{k}}{(2 \pi)^{9}} \frac{e^{i \vec{k} \vec{r}}}{|\vec{k}|^{2}}=\frac{15}{2(2 \pi)^{4}} \frac{1}{r^{7}},
$$

we obtain

$$
V_{0}(r)=\frac{1}{2 \pi R} \int \frac{d^{9} \vec{k}}{(2 \pi)^{9}} e^{i \vec{k} \vec{r}} A_{\text {tree }}=\frac{15}{16} \frac{N_{p} N_{s}}{R^{3} M_{\mathrm{Pl}}^{9}} \frac{v^{4}}{r^{7}} .
$$

In order to obtain this result we have included a normalization factor of $1 / \sqrt{2 E}$ for each state.

Using the above kinematical factor we can obtain the form of the one-loop contribution to the potential. The oneloop amplitude is given by

$$
A_{1 \text { loop }}=-\kappa_{11}^{4} c_{1} \frac{\pi^{3}}{384} M^{3} m^{3} v^{6}|\vec{k}|^{5} .
$$

We Fourier transform this expression using (3.6). Up to a constant the result for the one-loop contribution to the potential is then

$$
V_{1}(r) \propto N_{p} \frac{225}{64} \frac{N_{p}^{2} N_{s}}{R^{5} M_{\mathrm{Pl}}^{18}} \frac{v^{6}}{r^{14}} .
$$

We observe that the covariant amplitude differs by a factor $N_{p}$ from the result calculated in [2] using a classical picture. The difference is due to the fact that the covariant amplitude integrates over all eleven-dimensional momenta running in the loop while in the $\mathrm{M}$ (atrix) theory calculation only states with fixed $p_{-}$are allowed to run through the loop. Both amplitudes are related ${ }^{6}$ by a phase factor times $N_{p}$. This has to be taken into account when loop diagrams are calculated while tree diagrams naturally agree.

\section{QUANTUM GRAVITY CORRECTIONS}

$\mathrm{M}$ (atrix) theory not only allows us to compute corrections coming from general relativity but the much harder quantum gravity corrections. They have, in general, fractional powers of $\kappa$ because a dimensionful parameter, the cutoff, is present in the calculation. An example of a correction of this sort is the $v^{8} / r^{18}$ term appearing in $\mathrm{M}$ (atrix) theory at two loops. The result for this term in the effective potential can be easily obtained by a simple extension of the calculation performed in [17]

\footnotetext{
${ }^{6}$ We thank David Gross and Lenny Susskind for discussions on
} this. 


$$
S_{v^{8}}=\frac{3 \times 5^{2} \times 7^{2} \times 59}{2^{11}} \frac{N_{s}^{2} N_{p}}{R^{7} M^{24}} \frac{v^{8}}{r^{18}}
$$

Susskind conjectured that this term is related to a four graviton amplitude involving the $R^{4}$ vertex of $\mathrm{M}$ theory [3]. ${ }^{7}$ From the $M$ theory point of view the following is known. The leading term in the $\mathrm{M}$ theory effective action is the conventional Einstein term. However, there could be corrections coming from higher dimensional operators:

$$
R^{2}+R^{3}+R^{4}+\cdots
$$

Fradkin and Tseytlin [22] have shown that

$$
R^{m}=0, \quad \text { for } m=0,1,2,3
$$

at one loop. The one-loop correction to the effective action takes then the form:

$$
S \propto \int d^{11} x \sqrt{-g} \Lambda_{11}^{3} R^{4},
$$

where $\Lambda_{11}$ is a dimensionful cutoff. This cutoff can be determined from the type-IIA string theory, as was done in [23] and [24]. These authors decompactified the four-graviton amplitude of $d=9$ closed superstring theory to calculate the $R^{4}$ interaction in eleven dimensions:

$$
S=\frac{1}{2 \kappa_{11}^{2}} \int \sqrt{-g} R+\frac{c}{\kappa_{11}^{2 / 3}} \int \sqrt{-g} t_{8} t_{8} R^{4}
$$

where $c$ is a number that can be extracted from [23]. It is plausible to use this result to compute the analog of (4.1) from the supergravity point of view. While it seems hard to do a precise calculation because we expect polarizations and fermions to be relevant, it is not too hard to see that the four graviton amplitude involving the vertex (4.5) indeed gives a contribution to (4.1). We can use the probe-source picture of the previous sections. ${ }^{8}$ Higher-order local curvature invariants in eleven-dimensional supergravity do not change the form of $h_{--}$[25]. The only effect of such a term is to change the action of the probe, so that the equation of motion for $\varphi$ involves a new potential:

$$
V(\vec{x}, \tau) \varphi \propto\left(\frac{\partial^{2} h_{--}}{\partial \vec{x}^{2}}\right)^{2} \partial_{\tau}^{4} \varphi+\cdots
$$

This potential comes from the term $\left(R_{-r-r}\right)^{2}\left(R_{+i+j}\right)^{2}$ of the interaction vertex. The dots indicate other contributions in which we are not interested. Up to a constant the effective action from the supergravity calculation is

$$
S_{v^{8}} \propto \frac{N_{p}^{3} N_{s}^{2}}{R^{7} M_{\mathrm{Pl}}^{24}} \frac{v^{8}}{r^{18}}
$$

The dependences on $v, r N_{s}, R$, and $M_{\mathrm{Pl}}$ agree with (4.7), but we seem to find a disagreement for the dependence on $N_{p}$. A similar conclusion was obtained independently in

\footnotetext{
${ }^{7}$ Some discussion on this appeared in [18-21].
}

${ }^{8} \mathrm{We}$ thank Joe Polchinski for discussions on this.
[20]. While the $\mathrm{M}$ (atrix) theory result is linear in $N_{p}$, the M-theory result is proportional to $N_{p}^{3}$. It seems plausible that the origin of this discrepancy is again the fact that in the M-theory calculation states with eleven-dimensional momentum are running through the loops, while in the M(atrix) theory calculation these states have a fixed $p_{-}$. This changes the $N_{p}$ dependence of the result as we have seen in the previous sections. A more careful analysis including polarizations and fermions would have to be done to check if the numerical coefficient agrees. While the M(atrix) theory calculation was an easy extension of our previous calculation [17], the discrete light core quantization (DLCQ) M-theory counterpart seems to be much harder [26].

One may want to ask at this moment: why should the supergravity calculation agree with the M(atrix) theory calculation at all, especially because of the discrepancies found in the recent papers [27,28] or why does the relativistic correction come out correctly in the calculation performed in [2]? Probably the most natural explanation ${ }^{9}$ would be that the quantities computed in [27] and [28] receive corrections in the large $N$ limit, while the quantity computed in [2] might obey a nonrenormalization theorem that guarantees that the supergravity result is reproduced by $\mathrm{M}$ (atrix) theory for finite $N$. Our goal for the near future should then be to find out which quantities are not renormalized in the large $N$ limit so that they can be computed with finite $N \mathrm{M}$ (atrix) theory. Maybe an argument along the lines of [29] can be carried out here as well.

\section{ACKNOWLEDGMENTS}

We would like to thank M. Dine, W. Fischler, M. Green, D. Gross, J. Maldacena, J. Polchinski, A. Rajaraman, J. Schwarz, S. Shenker, and L. Susskind for useful discussions. This work was supported by the U.S. Department of Energy under Grant No. DE-FG03-92-ER40701.

\section{APPENDIX}

In this appendix we are going to compute explicitly the integral:

$$
I=\lim _{\epsilon \rightarrow 0} \int d^{9} \vec{p} \frac{1}{\left|\vec{p}_{f}-\vec{p}\right|^{2}} \frac{1}{\left|\vec{p}_{i}-\vec{p}\right|^{2}} \frac{1}{p^{2}-p_{f}^{2}-i \epsilon},
$$

where $p=|\vec{p}|$ and the same for $\vec{p}_{f}$. The above integrand can have poles when the denominator vanishes. In order to regularize them we introduce a "graviton mass" $\sigma$. At the end of the calculation we will be interested in the leading order behavior in terms of $\sigma$. Therefore we would like to compute the integral:

$$
I=\lim _{\epsilon \rightarrow 0} \int d^{9} \vec{p} \frac{1}{\left|\vec{p}_{f}-\vec{p}\right|^{2}+\sigma^{2}} \frac{1}{\left|\vec{p}_{i}-\vec{p}\right|^{2}+\sigma^{2}} \frac{1}{p^{2}-p_{f}^{2}-i \epsilon}
$$

to leading order in $\sigma$. First it is convenient to use the formula

\footnotetext{
${ }^{9}$ We thank Lenny Susskind for discussions on this.
} 


$$
\frac{1}{a b}=\frac{1}{2} \int_{-1}^{1} \frac{d z}{(a(1+z) / 2+b(1-z) / 2)^{2}},
$$

to write

$$
\frac{1}{\left|\vec{p}-\vec{p}_{f}\right|^{2}\left|\vec{p}-\vec{p}_{i}\right|^{2}}=-\frac{1}{4} \int_{-1}^{1} \frac{1}{\Lambda} \frac{\partial}{\partial \Lambda}\left[\frac{1}{(\vec{p}-\vec{P})^{2}+\Lambda^{2}}\right] d z
$$

where

$$
\begin{gathered}
\vec{P}=\frac{1}{2}\left[(1+z) \vec{p}_{f}+(1-z) \vec{p}_{i}\right], \\
\Lambda^{2}=\sigma^{2}+\frac{1}{4}\left(1-z^{2}\right) k^{2},
\end{gathered}
$$

and $\vec{q}=\vec{p}_{f}-\vec{p}_{i}$ is the momentum transfer. Using this identity $I$ can be written in the form

$$
I=-\frac{1}{4} \int_{-1}^{1} d z \frac{1}{\Lambda} \frac{\partial}{\partial \Lambda} \widetilde{I}
$$

with

$$
\widetilde{I}=\lim _{\epsilon \rightarrow 0} \int d^{9} \vec{p} \frac{1}{(\vec{p}-\vec{P})^{2}+\Lambda^{2}} \frac{1}{p^{2}-p_{f}^{2}-i \epsilon} .
$$

We will compute the real and the imaginary part of the above integral separately. In order to do this we use the identity

$$
\frac{1}{p^{2}-p_{f}^{2}-i \epsilon}=\text { P.P. }\left(\frac{1}{p^{2}-p_{f}^{2}}\right)+i \pi \delta\left(p^{2}-p_{f}^{2}\right),
$$

where P.P. denotes the principal part and $\delta$ is the Dirac delta function. We will first begin with the imaginary part. It is given by

$$
\begin{aligned}
\operatorname{Im} \tilde{I} & =\pi \int d^{9} \vec{p} \frac{\delta\left(p^{2}-p_{f}^{2}\right)}{(\vec{p}-\vec{P})^{2}+\Lambda^{2}} \\
& =-\frac{\pi^{4}}{12} \frac{p_{f}^{6}}{P} \log \left(\frac{\Lambda^{2}+\left(\vec{P}+\vec{p}_{f}\right)^{2}}{\Lambda^{2}+\left(\vec{P}-\vec{p}_{f}\right)^{2}}\right) .
\end{aligned}
$$

Inserting in (A6) we can get the result for the imaginary part of $I$ to leading order in $\sigma$ :

$$
\operatorname{Im} I=\frac{\pi^{5}}{6} \frac{p_{f}^{5}}{q^{2}} \log \left(\frac{\sigma^{2}}{q^{2}}\right)+\mathcal{O}\left(\sigma^{2}\right)
$$

The real part of $\widetilde{I}$ is

$$
\operatorname{Re} \widetilde{I}=\int d^{9} \vec{p} \frac{1}{(\vec{p}-\vec{P})^{2}+\Lambda^{2}} \text { P.P. }\left(\frac{1}{p^{2}-p_{f}^{2}}\right)
$$

to get

$$
\operatorname{Re} \widetilde{I}=\int_{0}^{\infty} d k \int d^{9} \vec{p} \frac{\sin k\left(p^{2}-p_{f}^{2}\right)}{(\vec{p}-\vec{P})^{2}+\Lambda^{2}} .
$$

This integral is straightforward to compute, just a bit lengthy. Here we are only going to write down the terms of $\operatorname{Re} I$ in which we are interested. These are the terms that in the region for small momentum transfer are nonanalytic. To leading order in $\sigma$ they are

$$
\operatorname{Re} I=\frac{\pi^{6}}{4} \frac{p_{f}^{4}}{\left(4 p_{f}^{2}-q^{2}\right)^{3}}|q|^{5}\left(1-\frac{q^{2}}{6 p_{f}^{2}}+\frac{q^{4}}{80 p_{f}^{4}}\right)+\cdots .
$$

The leading nonanalytic behavior of $I$ in terms of $\vec{q}$ is then given by

$$
I=\frac{\pi^{6}}{256} \frac{|q|^{5}}{p_{f}^{2}}+i \frac{\pi^{5}}{6} \frac{p_{f}^{5}}{q^{2}} \log \left(\frac{\sigma^{2}}{q^{2}}\right)+\cdots .
$$

[1] T. Banks, W. Fischler, S. H. Shenker, and L. Susskind, Phys. Rev. D 55, 5112 (1997).

[2] K. Becker, M. Becker, J. Polchinski, and A. Tseytlin, Phys. Rev. D 56, 3174 (1997).

[3] L. Susskind, Talk at Strings '97.

[4] M. B. Green, J. H. Schwarz, and L. Brink, Nucl. Phys. B198, 474 (1982).

[5] J. G. Russo and A. A. Tseytlin, Nucl. Phys. B508, 245 (1997).

[6] R. P. Feynman, Acta Phys. Pol. 24, 697 (1963).

[7] B. S. De Witt, Phys. Rev. D 162, 162 (1966).

[8] S. Sannan, Phys. Rev. D 34, 1749 (1986).

[9] P. C. Aichelburg and R. U. Sexl, Gen. Relativ. Gravit. 2, 303 (1971).

[10] L. Susskind, "Another Conjecture about M(atrix) Theory," Stanford Report No. SU-ITP-97-11, hep-th/9704080.

[11] D. Berenstein and R. Corrado, Phys. Lett. B 406, 37 (1997).

[12] R. P. Feynman and A. R. Hibbs, Quantum Mechanics and Path Integrals (McGraw-Hill, New York, 1965).

[13] A. I. Akhiezer and V. B. Berestetskii, Quantum Electrody- namic, Interscience Monographs and Texts in Physics and Astronomy (Interscience, New York, 1965), Vol. XI.

[14] S. Weinberg, Phys. Rev. 140, 516 (1965).

[15] J. F. Donoghue, Phys. Rev. Lett. 72, 2996 (1994).

[16] N. Sakai and Y. Tanii, Nucl. Phys. B287, 457 (1987).

[17] K. Becker and M. Becker, Nucl. Phys. B506, 48 (1997).

[18] P. Berglund and D. Minic, Phys. Lett. B 415, 122 (1997).

[19] M. Serone, "A Comment on the $R^{4}$ Coupling in M(atrix) Theory," Report No. UVA-WINS-WISK-97-13, hep-th/9711031.

[20] E. Keski-Vakkuri and P. Kraus, "Short Distance Contributions to Graviton-Graviton Scattering: Matrix Theory versus Supergravity,' Caltech Report No. CALT-68-2148, hep-th/9712013.

[21] V. Balasubramanian, R. Gopakumar, and F. Larsen, "Gauge Theory, Geometry and the Large $N$ Limit," Report No. HUTP-97/A095, hep-th/9712077.

[22] E. S. Fradkin and A. A. Tseytlin, Nucl. Phys. B227, 252 (1983). 
[23] M. B. Green and P. Vanhove, Phys. Lett. B 408, 122 (1997).

[24] M. B. Green, M. Gutperle, and P. Vanhove, Phys. Lett. B 409, 177 (1997).

[25] G. T. Horowitz and A. R. Steif, Phys. Rev. Lett. 64, 260 (1990).

[26] S. Hellerman and J. Polchinski, "Compactification in the Lightlike Limit," Report No. NSF-ITP-97-139, hep-th/9711037.
[27] M. Dine and A. Rajaraman, "Multigraviton Scattering in the Matrix Model,' Report No. SLAC-PUB-7676, hep-th/9710174.

[28] M. R. Douglas and H. Ooguri, "Why Matrix Theory is Hard," Report No. LBL-40889, hep-th/9710178.

[29] J. Maldacena, "The Large $N$ Limit of Superconformal Field Theories and Supergravity,', Report No. HUTP-98-A097, hep-th/9711200. 